\title{
Síntesis in-situ, caracterización, evaluación antibacterial y nanotoxicológica de cueros sintéticos de cloruro de polivinilo conteniendo nanopartículas de cobre (PVC/NPsCu$\left.{ }^{\circ}\right)$
}

\section{In-situ synthesis, characterization, antibacterial and nanotoxicological evaluation of synthetic polyvinyl chloride leather containing copper nanoparticles (PVC/NPsCu${ }^{\circ}$ )}

\author{
Robert Salazar ${ }^{1},{\text { Myshell Aquino', }{ }^{1} \text { ris Alvarez }}^{1}$ \\ 1Laboratorio de Química Cuántica y Nuevos Materiales para la Innovación Tecnológica. D.A. de Fisicoquímica., \\ Facultad de Química e Ing. Química, UNMSM. Ciudad Universitaria, Av. Venezuela s/n Cdra. 34, Lima 1-Perú
}

Recibido : 30/05/2017 Aceptado: 14/08/2017

\section{RESUMEN}

Agentes capaces de transmitir enfermedades como las bacterias, virus y hongos son los principales causantes de las infecciones intrahospitalarias $(\mathrm{IIH})$ en el mundo, esto ha motivado el desarrollo de los Nuevos Materiales basados en la Nanotecnología, donde en una rama de este se sintetizan nanocompósitos antibacteriales del tipo: Polímero/Metal Antibacterial, sintetizando superficies con gran actividad inhibidora de bacterias.

En el presente trabajo, se han generado nanocompósitos poliméricos antibacteriales, preparando el Policloruro de Vinilo (PVC) de resina en emulsión como plastisol y sintetizando nanopartículas de Cobre dentro de la matriz polimérica (in-situ). La descripción de la metodología se da con la síntesis de un precursor de Cobre para posterior dispersión mediante pulsos ultrasónicos, posterior estabilización y mezclado con los aditivos del PVC para posterior reducción química durante el gelificado de los plastisoles. El análisis espectral de los laminados de PVC/NPsCu por medio de Fluorescencia de Rayos $\mathrm{X}$ en Energía Dispersiva (FRX-ED), utilizando el software específico PyMca 4.7.4, revelan que la importancia del tiempo de gelificación del PVC guarda relación con el grado de reducción del Ácido Ascórbico (AA) sobre el Cu2+. Por otro lado, resultados del análisis por Espectroscopia Electrónica de Barrido (MEB) revelan que las dimensiones de las NPsCuo varían en un rango de 50 a $250 \mathrm{~nm}$. Los patrones de difracción de Rayos X (DR-X) evidencian la parcial cristalización polimérica del PVC y la presencia de Cuo. Se confirmó la actividad antibacteriana de los laminados de PVC/NPsCu por el método de difusión de disco sobre Eschericcia coli $0157: \mathrm{H}_{7}$; el halo de inhibición promedio resultó ser de 9,7 mm que implica un significativo efecto bactericida sobre la cepa mencionada. La viabilidad del desarrollo de estos nanocompósitos poliméricos se estudió por medio de un ensayo nanotoxicológico mediante el análisis citotóxico de los nanocompósitos de $\mathrm{PVC} / \mathrm{NPsCu}^{\circ}$ en células de sangre periférica humana, concluyendo que dicho efecto citotóxico sobre células sanas es menor al $8 \%$.

La viabilidad del desarrollo de estos nanocompósitos poliméricos se estudió por medio de un ensayo nanotoxicológico mediante el análisis citotóxico de los nanocompósitos de $\mathrm{PVC} / \mathrm{NPsCu}^{\circ}$ en células de sangre periférica humana, concluyendo que dicho efecto citotóxico sobre células sanas es menor al $8 \%$.

Palabras clave: PVC, infecciones intrahospitalarias, nanocompósitos, nanopartículas de cobre

\section{ABSTRACT}

Disease transmitting microorganisms, such as bacteria, viruses and fungi, are the main causes of hospital-acquired infections ( $\mathrm{HAl}$ ). This is the justification for the development of nanotechnology-based new materials and, in particular, for the synthesis of antibacterial nanocomposites of the type polymer / antibacterial metal, which create surfaces with a large bacteria inhibiting activity.

In the present work, antibacterial polymeric nanocomposites have been created by preparing polyvinyl chloride (PVC) from a resin emulsion as plastisol and synthesizing copper nanoparticles in-situ within the polymer matrix. The methodology of the process includes the synthesis of a copper precursor, its dispersion by ultrasonic pulses, its stabilization and mixing with other PVC additives, in order to obtain chemical reduction during the gelation of the

\footnotetext{
* Correspondencia:

E-mail: robertsalazarruiz@gmail.com,myshell.ap21@gmail.com,yrisalvarez.ygac@gmail.com
} 
plastisols. Analysis of the PVC / NPsCu laminates by Energy Dispersive X-ray Fluorescence (EDXRF), using the PyMca software 4.7.4, reveals that the importance of the PVC gelation time is related to the degree of reduction of ascorbic acid (AA) on Cu2 +. On the other hand, examination by Scanning Electron Microscopy (SEM) shows that the size of the NPsCuo varies between 50 and $250 \mathrm{~nm}$. Examination by X-ray Diffraction (XRD) shows the partial polymer crystallization of PVC and the presence of $\mathrm{Cu}^{\circ}$. The antibacterial activity of the PVC / NPsCu${ }^{\circ}$ laminates was confirmed by the disc diffusion method on Escherichia coli O157: $\mathrm{H7}$; the average zone of inhibition was $9.7 \mathrm{~mm}$, indicating a significant bactericidal effect on this strain. A nanotoxicological assay was carried out to evaluate the viability of the development of these polymeric nanocomposites. The cytotoxic analysis of the PVC / NPsCu nanocomposites in human peripheral blood cells concluded that the cytotoxic effect on healthy cells was less than $8 \%$.

Keywords: PVC, hospital-acquired infections, nanocomposites, copper nanoparticles

\section{INTRODUCCIÓN}

Desde hace muchos años, en el mundo ha existido la problemática de las Infecciones Intrahospitalarias, que han conllevado a vivir en un ambiente infeccioso acarreando una serie de enfermedades perjudiciales para la salud humana. La presencia constante de bacterias superficiales, son vectores de enfermedades nocivas para el hombre y animales, un ejemplo de esto se basa en las estadísticas referentes a enfermedades nosocomiales (intrahospitalarias) y focos infecciosos en el ámbito público. Para contrarrestar estos problemas, en las últimas décadas se han venido desarrollando nuevos materiales capaces de inhibir e inactivar a las bacterias causantes de enfermedades, entonces ha surgido la necesidad de contar con nuevos materiales capaces de aplacar, disminuir o menguar los problemas que antes no habían sido resueltos totalmente, pero que ahora están al alcance sólo de la inventiva del hombre ya que el desarrollo la ciencia y la tecnología hacen posible la síntesis y aplicación de estos, es por eso que los compósitos Polímero/Metal Antibacterial, han venido a ser objeto de estudio para aplicaciones biocidas.

Son contados los polímeros que sean capaces de adecuarse a cualquier tipo de aplicación, entre los más importantes tenemos al PVC, que es el termoplástico más utilizado y difundido en el mundo, debido a su versatilidad y compatibilidad con los aditivos (sustancias que mejoran sus propiedades físicas, mecánicas, térmicas, eléctricas, entre otros), proporcionando características específicas al producto final. [1]. Naturalmente el PVC ha sido la base para amplio desarrollo aplicativo, tal es el caso del desarrollo de patentes asociados a este tema.

Una revisión de las patentes en las últimas décadas revela en la Fig. 1 , donde se manifiesta el continuo y creciente empleo del PVC para aplicativos mayormente de uso social.

Entre las aplicaciones del PVC en el campo industrial tenemos a la patente US $4977201 \mathrm{~A}$ [2], que formula un plastisol de PVC con alta capacidad de adhesión a metales actuando como buen sellador. La patente WO 2008133839 A1 [3], desarrolla formulaciones en base a PVC, nanopartículas (NTC,
$\mathrm{TiO}_{2}, \mathrm{CaCO}_{3}$, etc.) y compuestos de madera (Pino, Cedro, Roble, Abeto y combinaciones). La patente EP 1432758 B1 [4], desarrolla el uso de PVC plastificado con ciertos ésteres para usarse en equipos médicos (bolsas de sangre, juguetes y materiales para contactos con alimentos).

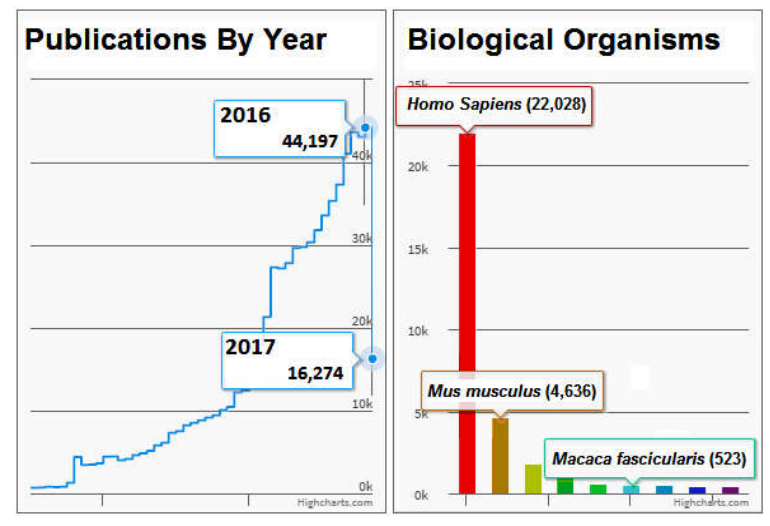

Figura. 1. Patentes de PVC en las últimas décadas [5]

En la figura 2, se representa el análisis en las últimas décadas de las patentes que emplean PVCantibacterial, las cuales llevan un ritmo creciente de estudios, desarrollo y aplicaciones médicas en organismos humanos, ensayos en ratones $y$ en primates.

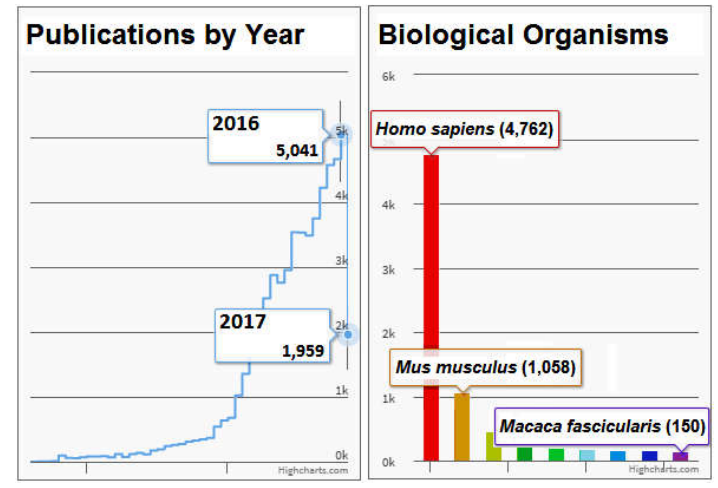

Figura 2. Patentes de PVC-Antibacterial en las últimas décadas [5] 
Como ejemplos tenemos a la patente US $5962137 \mathrm{~A}$ [6], que desarrolla un compósito aplicado a Tarjetas Antibacteriales en hospitales, donde al menos la capa frontal y posterior tiene un recubrimiento (PVC) y un agente antimicrobiano ( $\mathrm{Ag}, \mathrm{Cu}, \mathrm{Zn}$, sales cuaternarias de amonio) que inhiben la propagación de infecciones. La patente EP 0792654 B1 [7], forma un cuero plástico (PVC) con recubrimiento (Ag) como ayuda médica o quirúrgica para el tratamiento de infecciones en superficies de la piel (heridas) como profilaxis eficaz. La patente US 7799401 B2 [8], describe una lámina adhesiva y un método de fabricación con retardante de llama, esta lámina de PVC se una a una capa antibacteriana/desodorante (polvo metálico de Au, Ag y $\mathrm{Cu}$ ) para eliminar compuestos orgánicos volátiles (COV).

Por otro lado, es bien sabido que tanto el Cobre como sus aleaciones poseen cierta capacidad antibacterial $e$ inactivan a varios agentes microbianos potentes, incluyendo a Escherichia coli O157:H7, Listeria monocytogenes, Salmonella enteritis, Influenza A, entre otros [9]. Es por eso que el cobre se ha convertido en uno de los agentes biocidas más usados a nivel mundial, como material antiséptico, textiles bactericidas, pinturas antifouling, revestimientos intrahospitalarios, en agricultura, etc. Aspectos por las cuales son causas de investigación científica-industrial, volcando esfuerzos de investigación en el desarrollo de innumerables patentes con diversas aplicaciones; además, las áreas donde participa el cobre son diversas; Ciencia de los Materiales (29,8\%) es la principal rama científica, Química $(29,3 \%)$ y en tercer lugar Física y Astronomía (23,36\%) [10].

Los metales con capacidad antibacterial más reconocidos como la Plata, Cobre, Zinc, entre otros, aumentan dicha capacidad, si el tamaño de la partícula se va reduciendo, porque al ocurrir eso va aumentando el área superficial y por ende su reactividad; por ende, desde hace décadas se ha venido aprovechando el poder antibacterial de estos metales cuando se encuentran a nivel nanoparticulado, si bien la Plata supera al Cobre por su alta propiedad antibacterial, en este trabajo se empleará el Cobre por su bajo costo.

Varias son las teorías acerca de los mecanismos que expliquen la toxicidad de dichos metales, pero aún no esclarecen del todo, una de ellas explica que dependiendo de la propiedad del metal el comportamiento biocida se puede desencadenar por: (a) El potencial de reducción del metal. (b) La selectividad del átomo donante. El esquema 1, esquematiza mejor lo mencionado.

En (a) se encuentran los metales que tienen la suficiente capacidad para participar en reacciones redox y determinar la tendencia a adquirir electrones de un donante, estos metales redox activos actúan como cofactores catalíticos en muchas enzimas dentro de las células desencadenando la formación de ROS
(Especies reactivas de Oxígeno) generando un estrés oxidativo generando una muerte programada. Además, estos metales se asocian con procesos de respiración aerobia produciendo formas parcialmente reducidas de $\mathrm{O}_{2}, \mathrm{H}_{2} \mathrm{O}_{2}$ y superóxidos $\left(\mathrm{O}_{2}^{-}.\right)$, con la presencia de lagunas reacciones FENTON donde se intensifica la toxicidad del $\mathrm{O}_{2}$ por catalizar generando a la larga más estrés oxidativo.

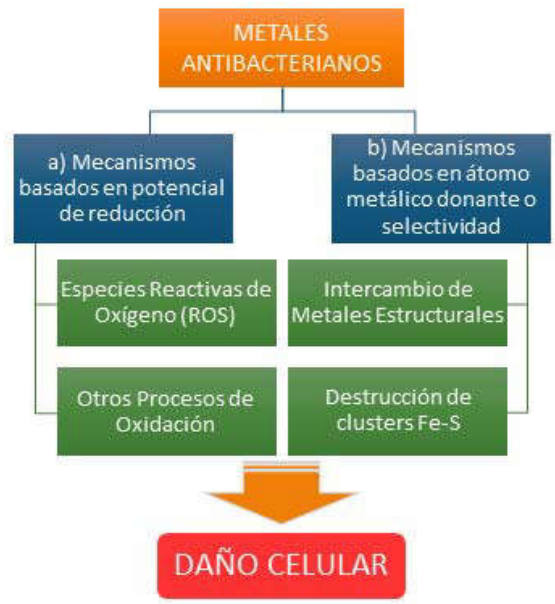

Esquema 1. Principales mecanismos que intervienen en la conducta antibacterial de los metales de acuerdo a la propiedad del metal responsable de la acción: (a) El potencial de Reducción y

(b) Selectividad del átomo donante.

En (b) se encuentran los iones metálicos que se unen a algunos átomos de ligandos donantes como $\mathrm{O}$, $\mathrm{N}$ y $\mathrm{S}$ a través de interacciones fuertes y selectivas. Es así que algunos iones metálicos pueden reemplazar a otros metales generando disfunciones celulares, fenómeno conocido como Mimetismo lónico o Mimetismo Molecular. Por ejemplo, estudios revelan que algunos metales (como el cobre) van a fomentar la destrucción de clusters expuestos [4Fe-4S] de las deshidratasas bacterianas en Eschericcia coli, que son muy susceptibles por metales tóxicos [11].

Los metales también pueden reemplazar a los metales de las uniones metálicas (centros no catalíticos) que inhiben la actividad enzimática, los iones cúpricos en particular llegan a formar complejos con grupos funcionales tales como Nitrógeno, Carbono u Oxígeno presentes en los microorganismos. Por último, se genera estrés oxidativo en algunas bacterias y levaduras expuestas a superficies de algunos metales [12]. Los logros al sintetizar NPsCu son muchos, empezando por métodos, siguiendo técnicas y eligiendo los reactivos y proporciones exactas para el logro de estos, pero no es muy fácil trabajar con el cobre por la característica química de poseer labilidad en sus electrones externos de las capas 3d y 4s, esa mínima energía de activación hace que el cobre pase de $\mathrm{Cu}^{+1} \mathrm{a} \mathrm{Cu}^{+2}$ como lo muestra la ecuación: 


$$
2 \mathrm{Cu}^{+} \stackrel{\Delta}{\leftrightarrow} \mathrm{Cu}^{0}+\mathrm{Cu}^{2+} \quad \mathrm{E}^{\circ}=+0.34 \mathrm{~V} \quad \ldots(1)
$$

Entonces el equilibrio de la reacción (1) puede desplazarse fácilmente en cualquier dirección, requiriendo una mínima energía de activación [13].

Entonces los logros de la síntesis de las NPsCu van a depender (para nuestro método de síntesis) siempre de la estabilización previo a la reducción del metal y del medio en el que se preparan las NPsCu.

Una forma conveniente de formar NPsCu se da cuando se logra la máxima dispersión de estas mediante la formación de complejos, una vía propuesta es la formación de carboxilatos metálicos, mediante reacción entre un Ácido Carboxílico y un ión metálico, como en la reacción [14]:

Ácido Graso $+\mathrm{Me}^{\mathrm{n}+}($ no alcalino $) \rightarrow$ Carboxilato metálico

Así, [15], ha sintetizado el complejo de Oleato de cobre, mediante la adición de una relación molar estequiométrica igual de $\mathrm{Cu}\left(\mathrm{NO}_{3}\right)_{2}$ con $\mathrm{NaOH}$ en solución y ácido oleico, el oleato de cobre formado es una pasta verdosa fácilmente identificable.

Otras maneras de sintetizar carboxilatos metálicos es lo que reporta [16], sintetizando una gran variedad de los mismos para posterior caracterización.

En este trabajo se obtuvieron complejos de cobre por reacción entre ácido oleico $(\mathrm{AO})$ y carbonato básico de cobre $(C B C u)$. Se usan diversos agentes estabilizantes o "capping" como la Polivinilpirrolidona (PVP) empleada por [17], empleando el PVP como regulador del tamaño y como agente protector del cobre (ver fig.3). Se sintetizaron NPsCu por dos metodologías, el primero empleando agua y el segundo empleando Etilenglicol, usando $\mathrm{CuSO} 4$ como precursor, AA como reductor y el PVP como estabilizante. De acuerdo con los análisis reportados el PVP actúa como agente polimérico y controlador de tamaño, creando así el compuesto complejo [ $\left.\mathrm{Cu}^{2+}-\mathrm{PVP}\right]$. Así se dificultan los núcleos de agregación a través de los grupos polares, que absorben fuertemente las partículas de cobre en la superficie con enlaces de coordinación. Los resultados mostraron que después de 8 horas en agua los tamaños de las partículas varían de $7 \pm 3 \mathrm{~nm}$ y $4 \pm 1$ $\mathrm{nm}$ cuando se agrega más PVP, mientras que en Etilenglicol por un tiempo de 1 hora las partículas son de $6 \pm 3 \mathrm{~nm}$ y $3 \pm 1 \mathrm{~nm}$ al usar más PVP.

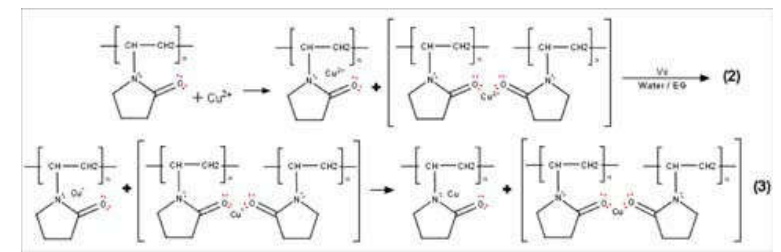

Figura 3. Ecuación de formación de complejo [ $\left.\mathrm{Cu}^{2+}-\mathrm{PVP}\right]$.
La capacidad reductora de algunos compuestos favorece la formación de las NPsCu, como los polioles, AA, NaHB4, Entre otros.

En el 2015, Mao [18] emplea un método de síntesis de NPs $\mathrm{Cu}$, empleando sulfato de cobre como precursor, hiposulfito de sodio como agente reductor y gelatina como agente de remate, la fase dispersante es el agua y no se hizo empleo de atmósferas protectora y a temperatura ambiente, se caracterizó la muestra en solución mediante UV, SEM, DRX, obteniéndose cuasiesferas y NPsCu con un tamaño aproximado de $32 \pm 3$ $\mathrm{nm}$. Otro logro importante se desarrolló al aprovechar las $\mathrm{NPsCu}$ como una solución rentable para la purificación de agua en filtros de papel. La metodología dice que se emplea $\mathrm{CuSO}_{4}$ y se añade $\mathrm{NaOH}$ para formar hidróxido de cobre, luego de un tiempo se forma $\left[\mathrm{Cu}(\mathrm{OH})_{2}\right] 2$ - para que se absorba en el papel y posterior reducción con Ácido Ascórbico (AA) (ver fig. 4,) a $85^{\circ} \mathrm{C}$ durante 10 a $30 \mathrm{~min}$. La caracterización respectiva (SEM, EDX) se observan nanopartículas de $12 \mathrm{~nm}$ de diámetro; además, estudios posteriores de análisis microbiológico sobre Escherichia coli, muestran que hay una gran inhibición de desarrollo de estas bacterias sobre el papel con NPsCu.

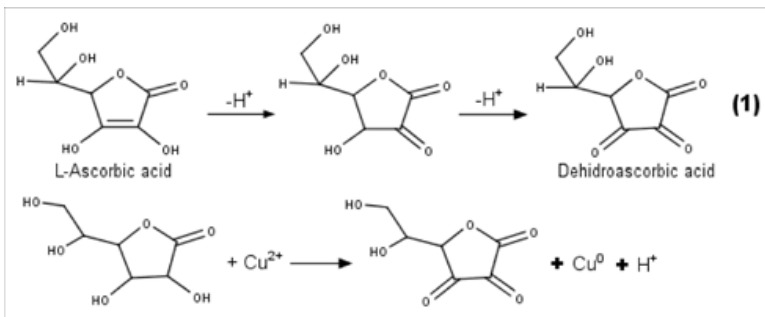

Figura 4. Ecuación y esquema de reacción de AA con $\mathrm{Cu}^{2+}$.

Ahora la tarea de aditivar las nanopartículas dentro de la matriz polimérica no resulta del todo fácil, pues las estabilidades de las mismas, las condiciones o el medio de formación de las NPs, deben ser capaces de poder acoplarse a la formulación y al proceso de obtención del polímero, es por eso que recurrimos al método de reducción química de manera in-situ, así aseguramos que la formación de las NPsCu se lleve a cabo dentro de la matriz polimérica.

Trabajos previos respaldan nuestra posición y metodología propuesta ya que existen síntesis y formación de nanocompósitos de Policloruro de Vinilo y nanopartículas metálicas por medios diferentes, Chen et al. (2004) [19], logran dispersar Fostato de Zirconio que contiene nanopartículas de plata sobre PVC, obteniendo muestras poliméricas con gran capacidad antibacteriana, empleando para esto un acoplador (Titanato orgánico) que mejora la compatibilidad interfacial PVC/polvo antibacterial, los resultados experimentales arrojaron excelentes propiedades antibacterianas, alcanzando casi el $100 \%$ de nivel 
bacteriostático con una carga del polvo antibacterial de 1.5 phr (por cien de resina).

Tamayo et al.[20], prepararon nanocompósitos poliméricos de Polietileno conteniendo cobre y plata, las nanopartículas de cobre de sintetizaron a partir de $\mathrm{CuCl}_{2}$ vía reducción con Citrato de Sodio y Borohidruro de Sodio en atmósfera inerte de Argón, la solución se torna de color café característico de las NPsCu; la síntesis del nanocompuesto de realiza mediante polimerización del eteno y posterior mezclado con las nanopartículas (sintetizados ex-situ). Estudios de capacidad antibacterial sobre Listeria monocytogenes corroboran el mecanismo de los iones $\mathrm{Cu}^{2+}$ sobre esta bacteria. Estudios de liberación de los iones por tiempos determinados se estudian mediante ICP, mientras que imágenes TEM revelan las nanopartículas penetran la pared celular y la membrana plasmática de las bacterias implicando cambios morfológicos sobre estas.

Rodríguez-Llamazares [21], prepara compuestos de $\mathrm{PVC} / \mathrm{CuO}$ y $\mathrm{PVC} / \mathrm{Cu}_{2} \mathrm{O}$ al $1 \%$ de los óxidos, mediante mezcla en fundido y llevados a extrusión obteniendo pellets, por moldeado para obtener láminas de $1 \mathrm{~cm} \times 1 \mathrm{~cm} \times 1 \mathrm{~mm}$ para análisis microbiológico, demostrando luego por medio de un mapeo por Microscopía Electrónica de Barrido (SEM) y EDS que el material compuesto se trata de un híbrido de óxido de cobre y polímero, estando las partículas dispersas uniformemente en la matriz polimérica. También se estudió la capacidad antibacterial de los óxidos de Cobre. Por otro lado, se estudió la adherencia de Escherichia coli ATCC 25922 en la superficie del material disminuye con respecto a una muestra de PVC puro, la reducción del tamaño a $30 \%$ de incubación de Escherichia coli demuestra que las superficies de PVC/ $\mathrm{Cu}_{2} \mathrm{O}$ inhiben el crecimiento de estas bacterias.

Además, Verde L. [22], funcionaliza la matriz polimérica de PVC (plastisol) con nanopartículas de Cobre obteniendo "nanocompósitos poliméricos". Estos se sintetizaron por dos métodos, el primero mediante la técnica de reducción química de una sal de Cobre $\left(\mathrm{CuAc}_{2}\right)$ dentro de la matriz polimérica (método in-situ), empleando Ácido Ascórbico como agente reductor y Polivinilpirrolidona como estabilizante. La segunda forma de obtención de NPs fue mediante la técnica de poliol, asistido con radiación microondas (20\% de potencia) usando $\mathrm{CuAc}_{2}$, AA y PVP (método exsitu). Luego se mezclan ambos y se le hace el curado a $180-200^{\circ} \mathrm{C}$. La caracterización vía FRX-ED y DR-X corroboran la presencia de Cuo en la matriz polimérica.

Por otro lado, es bien conocido que la presencia en exceso de metales como el Cobre, llega a ser tóxica a ciertos niveles de concentración y sobre ciertos organismos celulares donde intervienen las condiciones adecuadas para la liberación de iones y efectos antes mencionados. Es así que [23] diseña muestras poliméricas (Alginato y TEGMA/BisGMA, resinas en prótesis dentales) con NPsCu comercial $(5 \mathrm{~nm})$ con el fin de potenciar las prótesis para minimizar los riesgos de infecciones que actualmente poseen las mismas (representando el $45 \%$ de IIH en Chile el 2013), el ensayo toxicológico se desarrolla cultivando células sobre este para analizar el efecto de liberación del Cobre y posterior ensayo de toxicidad del mismo, los resultados concluyen que el polímero TEG/BisGMA es el principal causante de citotoxicidad sobre las células, descartando su uso en prótesis, y descartando la influencia del Cobre, se sugiere en este trabajo continuar la investigación con otros polímeros.

En conclusión, planteamos un método para la síntesis de $\mathrm{NPsCu}^{\circ}$ y dispersión en una matriz polimérica, generando nanocompósitos de $\mathrm{NPsCu}^{\circ}$ aprovechando los laminados como recubrimientosuperficie con capacidad antibacterial o beneficiarse de la formulación que emplea elementos de bajo coste, para el desarrollo de una variedad de productos.

\section{PARTE EXPERIMENTAL}

2.1 Dispersión del precursor junto con el plastificante DOP y el Ácido Oleico vía U.S.

El precursor de cobre se sintetizo en el laboratorio, mediante reacción entre $\mathrm{Na}_{2} \mathrm{CO}_{3}$ y $\mathrm{CuSO}_{4} \cdot 5 \mathrm{H}_{2} \mathrm{O}$ por dilución, mezclado con agitacion constante en caliente, filtrado, lavado con agua $\left(40{ }^{\circ} \mathrm{C}\right)$ y etanol absoluto, formando $\mathrm{CBCu}\left(\mathrm{Cu}_{2} \mathrm{CO}_{3}(\mathrm{OH})_{2}, \mathrm{PM}: 221.12 \mathrm{~g} / \mathrm{mol}\right)$ que sera empleado para formar el complejo oleato cúprico ( $\mathrm{Cu}[\mathrm{II}])$. Luego, para favorecer la dispersión y síntesis de nanopartículas metálicas se aplica la Técnica de Ultrasonido(Ultrasonic Processor, $20 \mathrm{KHz}, 600 \mathrm{~W}$, modelo GE600, punta ultrasónica CV17) [24] mediante pulsos ultrasónicos, con el fin de mejorar la homogeneidad y dispersión de los elementos que se agregan al PVC. Se dispersó CBCu sobre el dioctil ftalato (DOP) y se procedió al pulsado constantemente, a continuación se agregó AO; así se obtuvieron los complejos carboxílicos de cobre (II), se empleó luego PVP (SIGMA ALDRICH 40 KD) como agente dispersante, luego se agrega AA (MERCK) como agente reductor[22], manteniendo la relación molar $[\mathrm{AA} / \mathrm{Cu}]=2$ y se continuó pulsando constantemente manteniendo la temperatura por debajo de los $80^{\circ} \mathrm{C}$ para evitar la oxidación del cobre hasta observar que se llevó a cabo una reducción parcial del cobre (solución ámbar). La formulación del plastisol se presenta en la Tabla 1. 
Tabla 1. Insumos y cantidades empleadas

\begin{tabular}{|l|c|}
\hline \multicolumn{1}{|c|}{ INSUMO } & CANTIDAD \\
\hline Policloruro de vinilo (PVC) & 50 \\
D.O.P. & 25 \\
Estabilizante Lx-410 & 1 \\
${\text { Solvente }{ }^{\circ} \text { 3 }}^{\text {Carbonato básico de Cu (PM:221,12) }}$ & 1 \\
Ácido Oleico & 2 \\
Polivinilpirrolidona (PVP 40 KD) & 5 \\
Ácido Ascórbico (AA) & 5 \\
\hline Cu (\%) & 2 \\
PLASTIFICACIóN & $\mathbf{1}$ \\
[AA/Cu] (mol) & $\mathbf{5 0}$ \\
\hline
\end{tabular}

\subsection{Preparación del plastisol}

Luego de la previa dispersión, se mezcla el estabilizante (LX-410 Ba-Cd-Zn), y el solvente $\mathrm{N}^{\circ} 3$ (aguarrás mineral), mediante un mezclador de inmersión (solubilizador de Cowles) y un mezclador mecánico, variando la velocidad hasta $480 \mathrm{rpm}$ para que la mezcla se homogenice. Se informa que estos aditivos son tan bien utilizados en la industria de fabricación de cueros sintéticos.

\subsection{Gelificación del plastisol}

Se logran gelificar láminas del plastisol, agregando la mezcla de PVC sobre un papel siliconado especial (transfer) y se dispersa controlando el espesor del laminado final, la figura inferior nos deja ver una muestra antes (fig. 5(a)) y después (fig. 5(b)) de gelificar en estufa a $200^{\circ} \mathrm{C}$ a 2.5 minutos, se deja enfriar a la temperatura local.

Las muestras fueron comparadas mediante análisis FRX-ED con la misma temperatura a diferentes tiempos para muestras de PVC/NPsCuo para analizar el grado de reducción del $\mathrm{Cu}^{+2}$. Los laminados se exponen en la figura 5 .

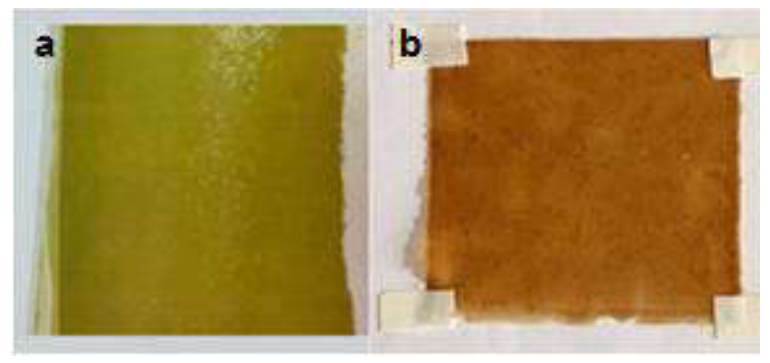

Figura 5. (a) Muestra previa a la gelificación de PVC. (b) Muestra gelificada de PVC por un tiempo de 2.5 minutos $a 200^{\circ} \mathrm{C}$, evidenciando la formación de NPS de $\mathrm{Cu}^{\circ}$.

\section{RESULTADOS Y DISCUSIÓN}

\subsection{Fluorescencia de Rayos X en Energía Dispersiva (FRX-ED)}

Se utilizó un espectrómetro de rayos $X$ con un Analizador Multicanal Amptek, con Tubo de Rayos $X$ con Ánodo de Ag y fuente de voltaje Eclipse III Amptek con rango de evaluación de 0-30 KeV y de intensidad 15 $\mu A$, el espectro resultante se observó con el programa específico DppMCA Versión 1.0.0.16, con previa calibración empleando una probeta de Acero inoxidable (SS316), los análisis de los datos espectrales se realizaron con el programa PyMca versión 4.7.4. La región de análisis fue de 3,5 - 10,83 Kev, con parámetros de ajuste: Chi Square 2,6572 y Last Chi square $0,345 \%$. El programa usa por defecto una base de datos incorporada con las energías correspondientes a cada elemento, donde se encuentran las energías de emisión de las capas K, L, M. La Tabla 2 muestra que la energía de emisión del cobre está cuantificada a $8,028,8,048$ y $8,90 \mathrm{Kev}$, con sus respectivas líneas de emisión características. En la figura 6, se analizan los espectros FRX-ED de una muestra respecto al grado de reducción del cobre con respecto al tiempo de gelificación, con el visualizador gráfico de OriginPro 2015, se ha tomado la región de Energía característica donde aparecen dos picos de cobre (8,04 y 8,90 Kev), estos fueron estudiados para tiempos de $0,5,1,2$ y 3 minutos, estableciendo el tiempo de gelificación óptimo de entre 2 a 3 min para futuros trabajos, ya que se desea evitar la degradación del PVC y en tiempos breves la reducción es mínima.

TABLA 2. Cuantificación de la energía de emisión del cobre. (PyMca)

\begin{tabular}{|c|c|c|}
\hline \multicolumn{3}{|c|}{ ENERGIES OF X-RAY EMISSION LINES } \\
\hline Line & Energy (KeV) & Rate \\
KL2 & 8,028 & 0,29913 \\
KL3 & 8,048 & 0,58282 \\
KM2 & 8,905 & 0,03989 \\
KM3 & 8,905 & 0,07816 \\
\hline
\end{tabular}

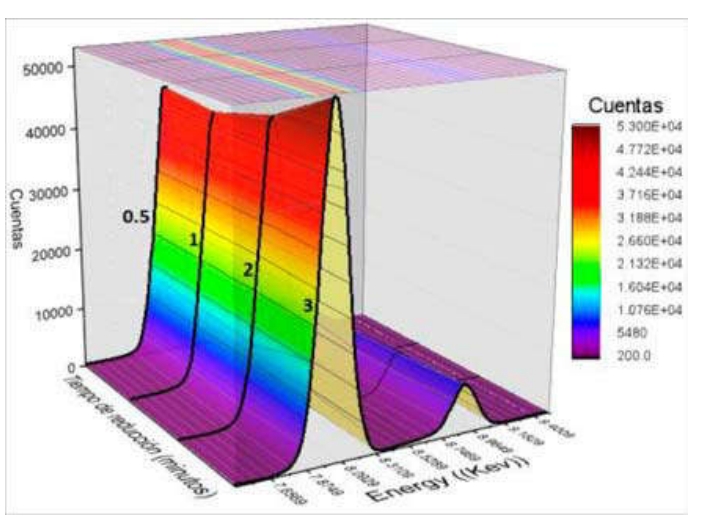

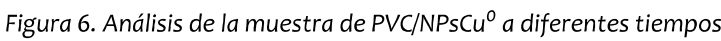
de gelificación. 
En la fig. 7, se muestra el espectro de FRX para una muestra de PVC-NPsCu, se determina la presencia de metales y no metales en la muestra, por Energías de Dispersión características de cada metal, se observan los picos preponderantes del Cloro (componente del PVC) y del Cobre metálico; además, más adelante, se determinan los picos de $\mathrm{Ba}, \mathrm{Cd}, \mathrm{Zn}$ procedentes del estabilizante del PVC; además del $\mathrm{Fe}, \mathrm{Ni}, \mathrm{Zn}, \mathrm{Cr}$ muy poco representativos.

Aquí, se desea resaltar que mediante el análisis espectral por medio del software PyMca, se obtiene la información de los elementos que conforman la muestra de PVC, tanto de los aditivos propios como de las nanopartículas formadas. No se ha tomado todo el rango espectral, no analizaremos al pico concerniente a la energía de Cloro; además, debido a la energía de emisión del equipo, no se está detectando elementos como el Carbono y Oxígeno, aquí no se estudiarán por su baja relevancia para nuestro análisis.

En la figura 8, se toma una porción del espectro para mayor claridad, en esta zona, mediante las deconvoluciones respectivas se determina el aporte energético de los átomos referentes al estabilizante metálico del PVC (LX-410 Zn-Ba-Cd), entre otros elementos traza que componen el PVC y/o de los reactivos empleados, refiriéndose al Calcio, Cromo y Hierro específicamente.

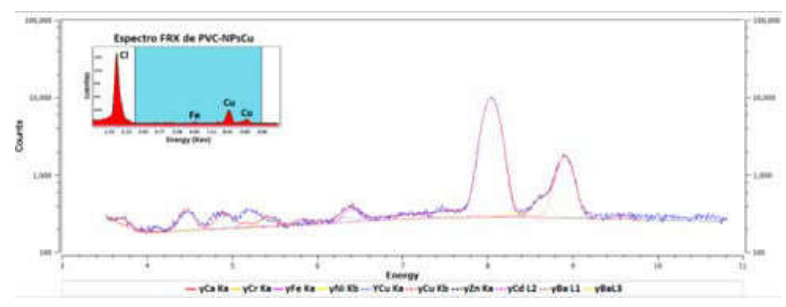

Figura 7. Espectro FRX de la muestra de PVC conteniendo NPsCu ${ }^{\circ}$.

La fig. 9, muestra la zona de mayor importancia para nuestro estudio, pues aquí viene resalta el espectro relativo a las energías de emisión del cobre, se observan bandas acopladas en las zonas de energías correspondientes de la capa $\mathrm{Ka}(\mathrm{KL2}=8,028$ y KL3 $=8,048$ $\mathrm{Kev})$ y la capa $\mathrm{Kb}\left(\mathrm{KM}_{2}=8,905\right.$ y $\left.\mathrm{KM} 3=8,905 \mathrm{Kev}\right)$, aunque el análisis más exhaustivo y detallado para este elemento deberán reportar picos propios de contribución empleando equipos de mayor precisión, aun así los reportes obtenidos por ahora son de gran ayuda. Aportes de otros metales como el $\mathrm{Ni}$ y el $\mathrm{Zn}$ (propio del estabilizante), se confirman mediante el análisis por medio del software mencionado.

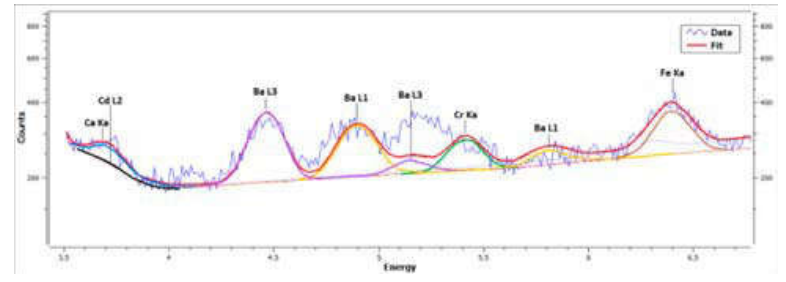

Figura 8. Determinación de elementos que componen los aditivos del PVC.

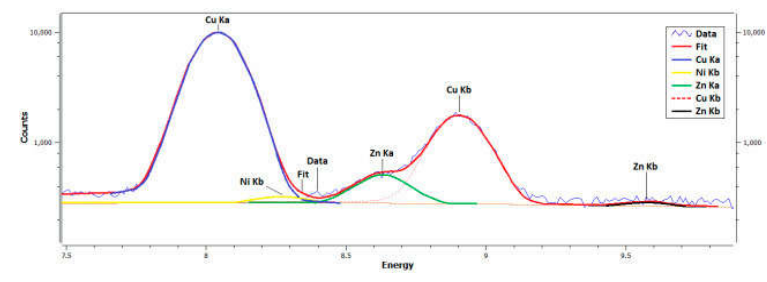

Figura 9. Determinación de Cobre en la muestra de PVC analizada

\subsection{Espectroscopía FT-IR}

La fig. 10 muestra el espectro de IR del PVC conteniendo $\mathrm{NPsCu}^{\circ}$ comparado con el espectro patrón de una base de datos (NICODOM IR Polymers) del equipo con un $85,80 \%$ de probabilidad, se observa una pequeña banda de absorción a $1581 \mathrm{~cm}^{-1}$, se asigna este valor para la vibración de tensión del doble enlace $\mathrm{C}=\mathrm{C}$, formado por la dehidrocloración del polímero debido a la degradación por temperatura; además, se reconocen las bandas de absorción para el PVC muy similares a lo que reportan en otros estudios. Otras bandas de absorción características son: $694 \mathrm{~cm}^{-1}$ para el estiramiento $\mathrm{C}-\mathrm{Cl}, 833 \mathrm{~cm}^{-1}$ para el estiramiento de la cadena, $1257 \mathrm{~cm}^{-1}$ para la flexión $\mathrm{C}-\mathrm{H}$ en el plano, 1336 $\mathrm{cm}-1$ flexión $\mathrm{C}-\mathrm{H}$ fuera del plano (hombro), $1427 \mathrm{~cm}^{-1}$ flexión en el plano del $\mathrm{CH}_{2}, 2854 \mathrm{~cm}^{-1}$ estiramiento simétrico en plano $\mathrm{CH}_{2}$, entre otros.

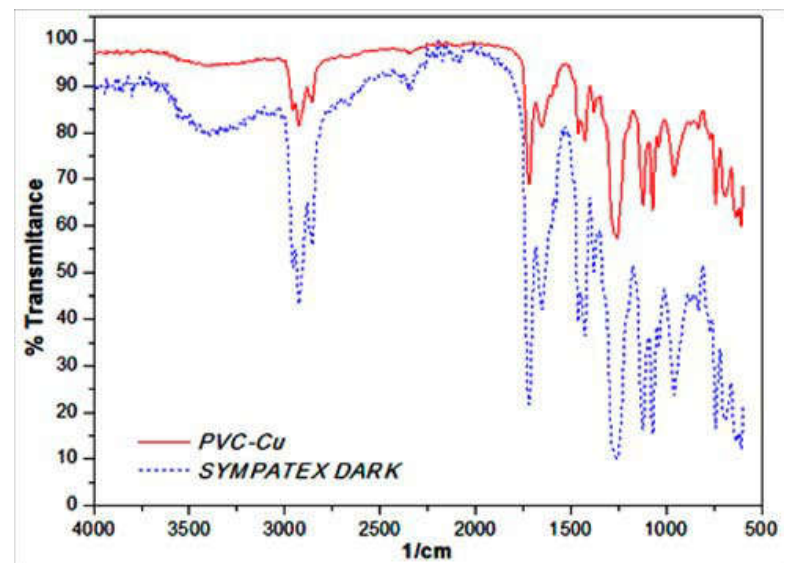

Figura 10. Espectro Infrarrojo por ATR del nanocompósito PVC/NPsCu ${ }^{\circ}$, comparado con espectro SYMPATEX DARK de la base espectral NICODOM.IR. 


\subsection{Microscopía Electrónica de Barrido (MEB)}

La dispersión de las $\mathrm{NPsCu}^{0}$ dispersadas en la matriz de PVC, fueron evaluadas con el microscopio MEB FEI INSPECT S50, operado a 30kv y trabajando a alto vacío.

Siguiendo la reducción del cobre por el ácido ascórbico, las imágenes MEB muestran la dispersión y el tamaño de las nanopartículas dispersas en el PVC. Se observa una buena dispersión de las partículas de diferentes diámetros debido a estabilización previa por parte del Ácido Oleico y el PVP (figura 11 a), y en lo que respecta al tamaño, se observa pocos aglomerados de partículas pequeñas de cobre (figura 11 a, b). El tamaño de las partículas obtenidas varía de 50 a $250 \mathrm{~nm}$ de diámetro, con un tamaño promedio de $152 \mathrm{~nm}$ (figura 11 c). Una densidad de pico EDS (figura 11 d) confirma la formación de cobre en la matriz polimérica al observar una intensidad a $8.04 \mathrm{Kev}$, típica energía para la línea Ka de $\mathrm{Cu}$, que es consistente con resultados de DRX más adelante.

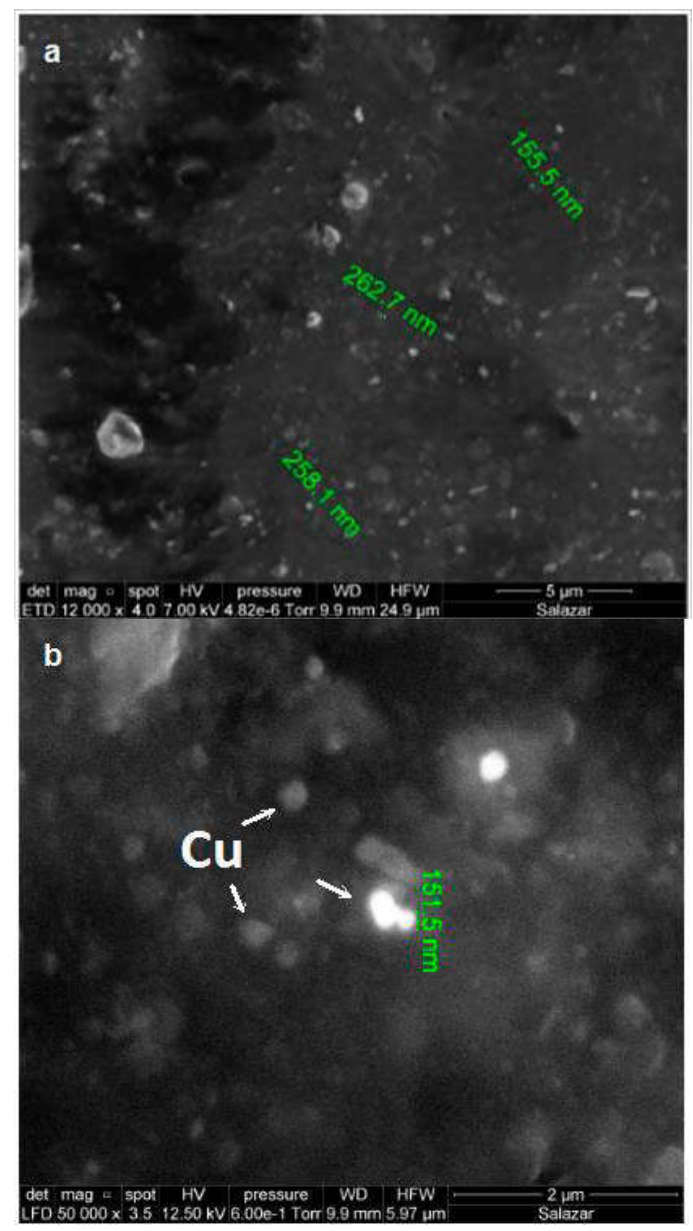

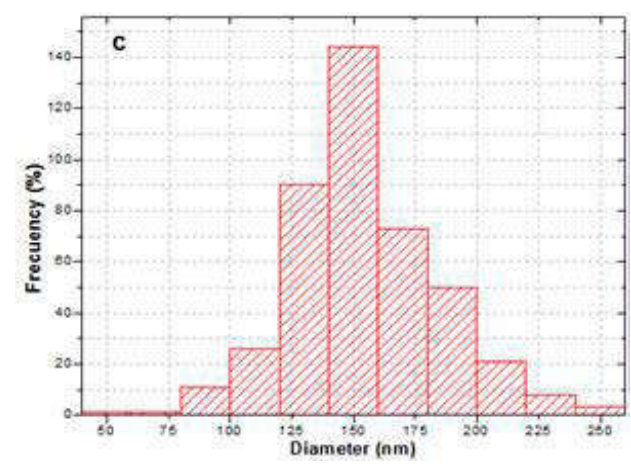

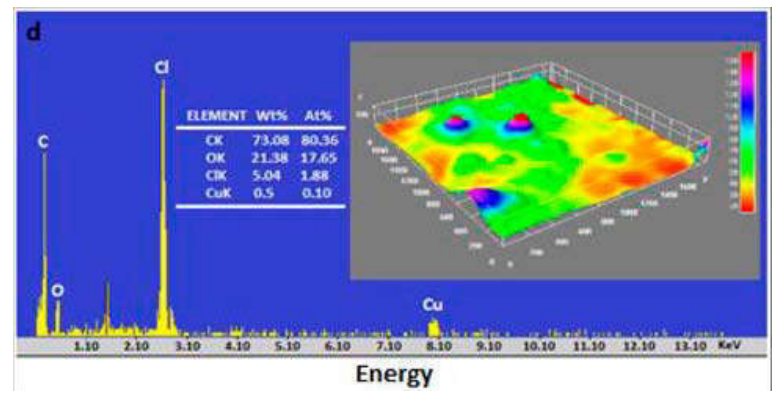

Figura 11. Caracterización de nanopartículas de cobre en la matriz polimérica de PVC: (a) $5 \mu m$ y (b) $2 \mu m$ de magnificación. (c) Muestra el histograma de distribución de tamaños de las nanopartículas por tratamiento de imagen. (d) Muestra el espectro EDS de nuestro nanocompósito y una vista superficial del área estudiada.

\subsection{Difracción de Rayos X (DR-X)}

La determinación de las fases de cobre en los laminados de $5 \mathrm{~cm} \times 5 \mathrm{~cm} \times 0.15 \mathrm{~mm}$, fueron analizados en un difractómetro marca Bruker, modelo D8-Focus, detector PSD Lynxeye, fuente de Radiación Cu Ka con una longitud de radiación de Rayos- $X$ de 1,5406 Å, en un barrido $2 \theta$ de $4-80^{\circ}$ y paso de 0,02 grados.

El difractograma de la fig. 12, corresponde a la muestra PVC/NPsCu y se observa un porcentaje de cristalización, con ángulos homólogos semejantes a los reportados [25], para ángulos inferiores a 34,36 , incluso estos datos presentan una variabilidad en función al tipo de resina de PVC utilizado, método de preparación de la muestra, temperatura de trabajo, entre otras variables y delimitan un grado de cristalización para muestras de la misma matriz de PVC entre 10 al $30 \%$ [26].

Estudios realizados en estos temas indican que existen mecanismos para la formación de nanocristales, se menciona en un ejemplo que, en ciertas condiciones, las fases se separan y se asocian a un sistema "espinoidal", donde aparecen dos regiones, una donde se agrupan las laminillas en porciones para poder cristalizar y la otra que es la parte amorfa del polímero, debido a que normalmente los polímeros poseen cierto orden de "cristalinidad" para describir el grado del orden dentro del mismo, es así que existen dos fases, la 
cristalina y la amorfa, las cuales se presentan en varios polímeros, incluido el PVC.

Además, en el difractograma de la figura 11, se observan dos picos característicos de las señales del cobre (0), aun cuando los picos característicos de una señal patrón del cobre (0) indican planos cristalinos correspondiente a las reflexiones de Bragg (HKL) de (111), (200) y (220) con ángulos de difracción $2(\theta)=43,37^{\circ}$, $50,53^{\circ}$ y $74,12^{\circ}$, el difractograma del compósito desarrolla ángulos de difracción $2(\theta)=42,50^{\circ}$ y $51,30^{\circ}$ con desviaciones de estas señales de $0,87^{\circ}$ y $0,77^{\circ}$ respectivamente. Cabe mencionar, que el estudio correspondiente de las desviaciones de estas señales no es el objetivo en este trabajo.

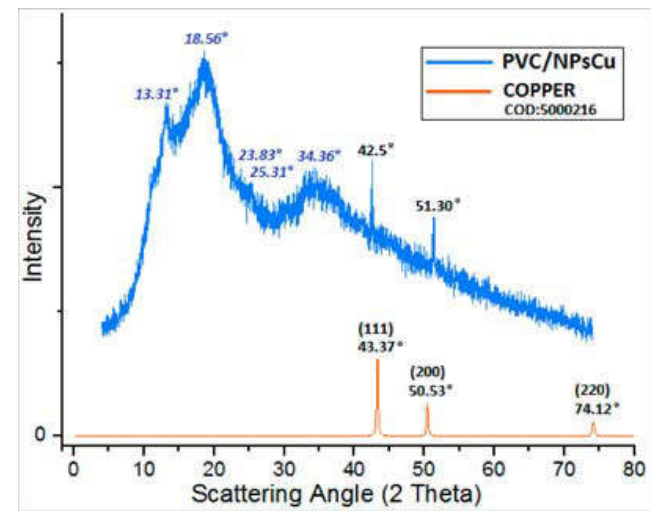

Figura 12. Patrón de difracción de la muestra obtenida de PVC/NPsCu y patrón de difracción de Cuo.

\subsection{Efecto bactericida de los laminados PVC/NPsCuo}

Utilizando el método de difusión de disco para antibióticos, se logró determinar la actividad antibacteriana de los laminados de PVC/NPsCu'. Se preparó un inóculo (Escherichia coli O157:H7) de 108 UFC/ml cuya absorbancia es de entre 0,08-0,1. El inóculo se sembró en placas Petri con agar Müller-Hilton. Una vez endurecido el medio de cultivo se colocaron discos de $5 \mathrm{~mm}$ de diámetro del nanocompósito. Se incubaron durante 24 horas a $37^{\circ} \mathrm{C}$ y se determinó el halo de inhibición alrededor del disco utilizando un vernier. Se utilizaron como controles negativos cultivos sin discos del material y se hicieron tres ensayos por triplicado de manera independiente. La Tabla 3 muestra los resultados, donde el halo de inhibición promedio es de $9.7 \mathrm{~mm}$ (cer fig. 13), mientras que la literatura reporta que Escherichia coli ATCC 25922 contra Ampicilina resulta un halo de $16-22 \mathrm{~mm}$ [27]. Este desnivel podría contrarrestarse con el aumento de concentración de $\mathrm{Cu}^{\circ}$ en la muestra. La figura 14 ilustra mejor los ensayos.
TABLA 3. Diámetros de la zona de inhibición bacteriana del nanocompósito

\begin{tabular}{|c|c|c|c|}
\hline ENSAYOS & 1 & 2 & 3 \\
\hline \multirow{3}{*}{ Diámetros de inhibición $(\mathrm{mm})$} & 9 & 9.7 & 10.1 \\
& 9.3 & 10.2 & 9.6 \\
& 10.4 & 9.8 & 9.5 \\
\hline
\end{tabular}

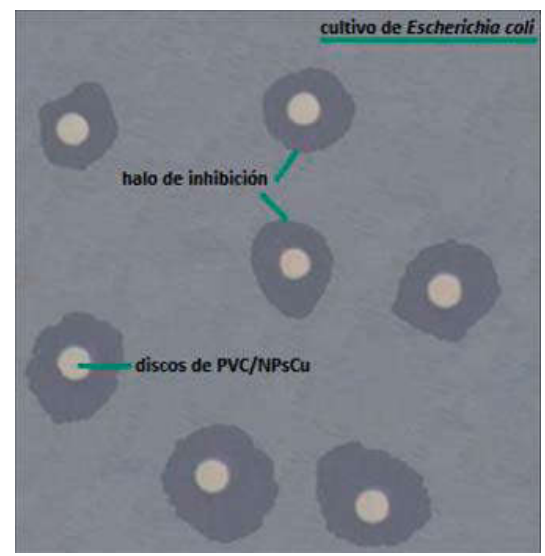

Figura 13. Imagen representativa de la actividad antibacteriana del nanocompósito PVC/NPsCuo, que generan halos de inhibición para Escherichia coli

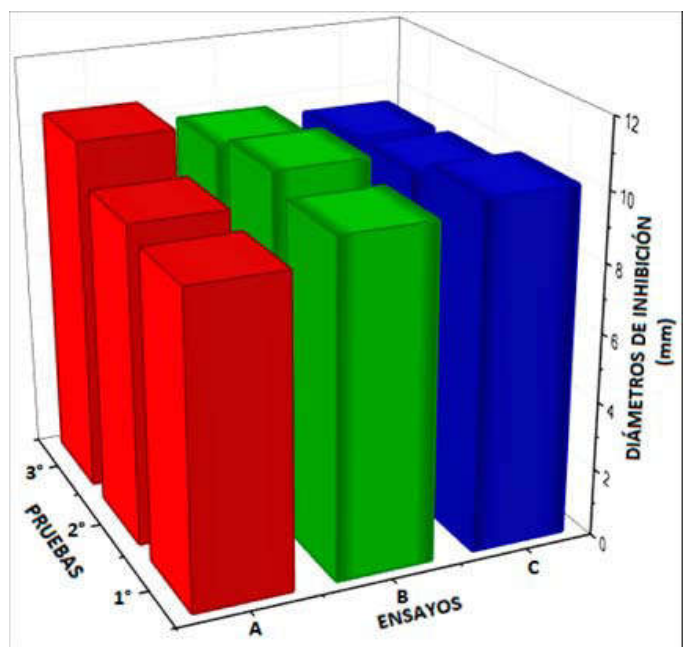

Figura 14. Diagrama que muestra la similitud de resultado en los ensayos.

\subsection{Estudio Nanotoxicológico de los laminados PVC/NPsCu ${ }^{\circ}$}

Con el fin de determinar el efecto citotóxico de los laminados de $\mathrm{PVC} / \mathrm{NPsCu}^{\circ}$, se aislaron células de sangre periférica humana utilizando un gradiente de Ficoll. Se sembraron las células en una caja Petri cuya superficie fue cubierta con un disco de $10 \mathrm{~cm}$ del material proporcionado. Las células se cultivaron durante 2 horas a $37^{\circ} \mathrm{C}$ con $5 \%$ de $\mathrm{CO}_{2}$ en un medio de cultivo RMPI (Roswell Park Memorial Institute) 1640 suplementado con $10 \%$ de suero bovino fetal y $1 \%$ de antibiótico. Una 
vez transcurridas estas 2 horas, se cambió el medio de cultivo por medio fresco, se despegaron los monocitos de los laminados de PVC/NPsCu y se ajustó el número de células a $20000 / \mathrm{ml}$ de medio.

Se resembraron en la caja con superficie del nanocompósito y se incubaron durante 72 horas. Se determinó la viabilidad de las células utilizando el colorante de exclusión vital Azul Tripano. Para tal efecto, se retiró el medio de cultivo y se lavaron las células 3 veces con buffer fosfato salino (PBS) $1 X$, posteriormente se tripsinizaron, se lavaron, tiñeron $y$ contaron. Como control negativo se utilizó un cultivo en el que no se colocó el laminado antibacterial en el fondo de la caja. Como control positivo se utilizó KCN cuya alta toxicidad es conocida. Se realizaron 3 ensayos independientes por triplicado.

TABLA 4. Diámetros de la zona de inhibición bacteriana del nanocompósito

\begin{tabular}{|c|c|c|c|}
\hline Ensayo & \multicolumn{3}{|c|}{ Número de células/ml de medio } \\
\hline 1 & $18750(1200)$ & $18962(986) 18$ & $18697(786)$ \\
2 & $18820(950)$ & $540(1142)$ & $18722(1057)$ \\
3 & $18635(1036)$ & $18956(924)$ & $18825(1103)$ \\
Control + & $345(19200)$ & $357(18926)$ & $335(19130)$ \\
\hline
\end{tabular}

En la Tabla 4 los números entre paréntesis representan las células muertas y los números fuera del paréntesis de las células vivas. Debido a que el número de células se ajustó a 20 000/ml de medio, las células faltantes son aquellas que se lisaron en las 72 horas de tratamiento. En general se observó un efecto bactericida significativo mientras que, el efecto sobre células sanas fue siempre menor al $8 \%$. La figura 15 ilustra mejor los ensayos.

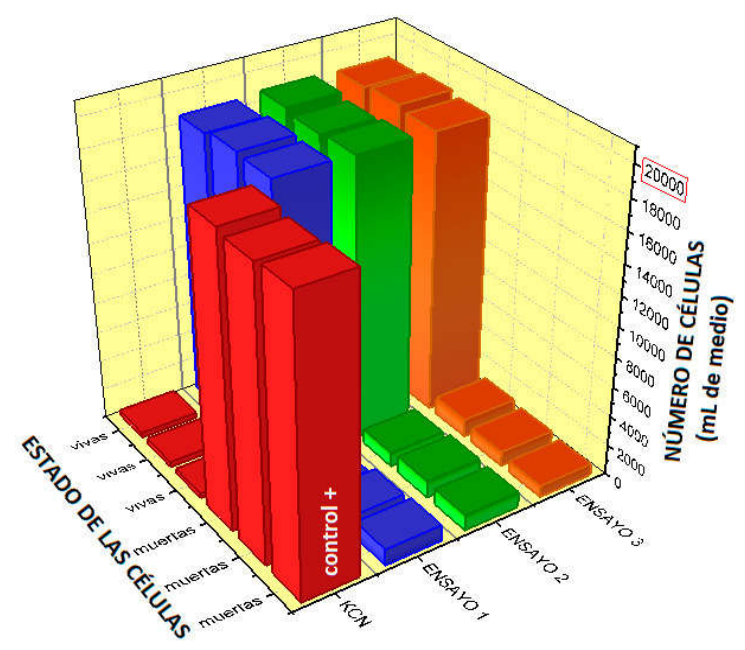

Figura 15. Representación gráfica del estudio citotóxico de los laminados de PVC/NPsCu en sangre periférica humana, concluyendo que el efecto citotóxico en menor al $8 \%$.

\section{CONCLUSIONES}

Se lograron sintetizar los laminados de PVCl $\mathrm{NPsCu}^{\circ}$ con el método y las secuencias mostradas, logrando emplear CBCu como un agregado al PVC, la formulación que empleamos presenta un buen balance general de aditivo y en lo que respecta al ácido ascórbico, éste logra la reducción del cobre, evidenciándose primero en el cambio de color del plastisol al ser tratado térmicamente y el análisis del espectro de Fluorescencia de Rayos $X$ en energía dispersiva, corroboraron: la presencia del $\mathrm{Cu}^{\circ}$, la presencia de otros elementos y ayuda a determinar que el tiempo de gelificación es un parámetro clave para lograr la reducción completa del cobre, aumentando este a medida que aumenta el tiempo de curado. Las micrografías MEB mostraron un panorama global acerca de la distribución de tamaño de nuestras nanopartículas sintetizadas, no se pudo observar de manera más detallada la morfología de las NPs debido a la degradación del PVC en las condiciones sometidas.

La similitud de los espectros FTIR, evidencia que las muestras obtenidas no presentan degradación del material y/o grupos funcionales por que las temperaturas alcanzadas no lo permiten ya que la formulación es óptima; además, la temperatura Flash Point del PVC es de $390^{\circ} \mathrm{C}$. Los análisis de Difracción de Rayos $\mathrm{X}$, nos muestran también picos característicos que revelan la presencia del cobre en la muestra de PVC.

Los resultados del análisis del Efecto Bactericida del laminado de PVC/NPsCu ${ }^{\circ}$ son favorables demostrando la viabilidad de desarrollar compósitos con potencial antibacteriano, los resultados no son desalentadores puesto que se puede aumentar la concentración del cobre en la muestra ya que la muestra contaba con una concentración del $1 \%$ de cobre.

El Ensayo Nanotoxicológico también arroja buenos resultados reforzando la intensión de emplear la formulación para desarrollar productos antibacteriales de PVC.

\section{AGRADECIMIENTOS}

Los autores expresan su agradecimiento al FINCYT por la financiación a través del Proyecto N¹35-FINCyTIB-2013. Al Instituto Tecnológico de la Producción del Ministerio de la Producción por la provisión de sus laboratorios para la parte experimental.

Especial agradecimiento al Quím. Aldo Javier Guzman Duxtan, profesor de la Facultad de Química e Ingeniería Química de la UNMSM por el asesoramiento en el tema desarrollado y a la Dra. Yanis Toledano Magaña del Centro de Nanociencias y Nanotecnología de la UNAM, por el apoyo brindado en los análisis antibacteriales, citotóxicos de las muestras. 


\section{GLOSARIO DE TÉRMINOS}

Policloruro de vinilo, Antibacterial, Nanopartículas de Cobre, Plastisoles, Reducción Química, PyMca 4.7.4.

\section{REFERENCIAS}

[1] M. I. Beltran, "Los procesos de gelificación y descomposición de los plastisoles de PVC por FTIR y TG. La influencia del tipo de resina, plastificante, composición y otras variables", tesis doctoral, Universidad de Alicante, Alicante, España, 1995.

[2] A. Ogawa, M. Shimada, K. Ando, "Polyvinyl chloride plastisol composition", US 4977201 A, 1990, Dic. 11.

[3] L. Matuana, "Hybrid PVC/WOOD-reinforcement nanocomposites and Method of manufacture", WO 2008133839 A1, 2008, Nov. 6.

[4] C. Gosse, P. Daniels, T. Larson, "Plasticised polyvinyl chloride", EP 1432758 B1, 2008, Nov. 26.

[5] (2017) The LENS website. [Online]. Available: https:// www.lens.org/lens/

[6] T. Ochiai, S. Ito, "Antibacterial Card", US 5962137 A, 1999, Oct. 5.

[7] J. M. Geb, G. Bähr, "Articles with antibacterial activity for use as medical or surgical aids", EP 0792654 B1, 2001, Oct. 17.

[8] D. Lee, M. OK, D. Lim, "Adhesive sheet and manufacturing method thereof", US 7799401 B2, 2010, Sept. 21.

[9] ProCobre, "Cobre: Salud, Medio Ambiente y Nuevas Tecnologías", 2014, [En línea]. Disponible en: http:// procobre.org, [accesado el 15 de mayo del 2015].

[10] A. Guzmán, R. Salazar, "Boletín de Vigilancia Tecnológica: El Cobre I+D+i”, UNMSM, PERÚ, 2016 [en línea]. Disponible en: https://www.researchgate.net/publication/ 308961689_Boletin_de_Vigilancia_Tecnologica_El_Cobre_IDi_2 016 UNMSM-PERU [Accedido 15-Julio-2016]

[11] F. Fang Xu, J. Imlay, "Silver(I), Mercury(II), Cadmium(II), and Zinc(II) -target Exposed Enzymic Iron-Sulfur Clusters when They Toxify Escherichia coli”, Appl. Environ. Microbiol. Vol. 78, no. 10, pp. 3614-3621, Feb. 2012.

[12] H. Palza, "Antimicrobial Polymers with Metal Nanoparticles", Int. J. Mol. Sci. Vol. 16, pp. 2099-2116, Jan. 2015

[13] K. Delgado Vargas, "Estudio de la obtención de compósitos con propiedades Antimicrobiales y Antifouling formados por una matriz polimérica y nanopartículas a base de cobre", tesis doctoral, Universidad de Chile, Santiago de Chile, Chile, 2013.

[14] "Síntesis y Caracterización de Carboxilatos Metálicos", G. Cerillo, [en línea], Disponible en: https://upcommons.upc.edu/ bitstream/handle/2099.1/6843/Resum.pdf

[15] M. Song, Z. Zhang, "A simple way to prepare Cupric oleate capped Agl nanoparticles", Mat. Resch, Bol., Vol. 39, pp. 22732278, Jul. 2004.

[16] E. F. Ardiles, "Caracterización Magneto-Estructural de compósitos de Cobre (II)-carboxilatos", tesis para optar el título de Químico, Universidad de Chile, Santiago de Chile, Chile, 2010.

[17] W. Yu, H. Xie, L. Chen, "Synthesis and Characterization of Monodispersed Copper Colloids in Polar Solvents", Nanoscale Res. Lett. Vol. 4, pp. 465-470, 2009.

[18] A. Mao, M. Ding, X. Jin, X. Gu, C.Cai, C. Xin, T. Zhang, "Direct, rapid synthesis of wáter-dispersed copper naoparticles and their surface-enhanced Raman scattering properties", J. Mol. Struc., pp. 396-401, 2015

[19] X. Chen, C. Li, L. Zhang, S. Xu, Q. Zhou, Y. Zhu, X. Qu, "Main factors in preparation of antibacterial particles/PVC composite", China Particuology, Vol. 2, no. 5, pp. 226-229, 2004.

[20] L. Tamayo, M. Azócar, M. Kogan, A. Riveros, M. Páez, "Copperpolymer nanocomposites: An ezcellent and cost-effective biocide for use on antibacterial surfaces", Mater. Sci \& Eng., (2016)

[21] S. Rodríguez, M. Mondaca, C. Badilla, A. Maldonado, "PVC/ Copper oxide composites and their effect on bacterial adherence", J. Chil. Chem Soc., Vol. 57, no. 2, pp. 1163-1165, 2012.

[22] A. Guzman, L. Verde, J. Rengifo, "Synthesis and characterization of copper nanoparticles/polyvinyl chloride (CuNPs/PVC) nanoparticles", Proc. Mater. Sci., col. 9, pp. 298304,2015 .
[23] N. Galarce Toro, "Efecto de nanopartículas de cobre sobre la viabilidad celular en compósitos poliméricos", tesis para optar el título de Ing. Civil Química, Universidad de Chile, Santiago de Chile, Chile, 2013.

[24] D. hen, S. Sharma, A. Mudhoo, Handbook of Applications of Ultrasound Sonochemistry for Sustainability, FL, USA: CRC Press, 2012

[25] S. Guerrero, H. Veloso, "On the analysis of wide-angle X-ray diffraction curves of Poly(vinyl chloride) samples", Pol., Vol. 31, pp. 1615-1622, 1990.

[26] R. Chartoff, T. Lo, E. Ray, H. JR, R. Joon -roe, "Infrared spectral changes with crystallization in poly(vinylchloride): Correlations with X-ray and density data", J. Macr. Sci. Physics, Vol. 20, no. 3, pp. 287-303, 2006.

[27] MINSA Manual de procedimiento para la prueba de sensibilidad antimicrobiana por el Método de Disco de Difusión, Serie de Normas Técnicas $\mathrm{N}^{\circ} 30$. Disponible en: http://190.102.152.73/ repositorioaps/o/4/jer/-1/manua |\%2osensibilidad.pdf, [Accesdido:20-jun-2017]. 